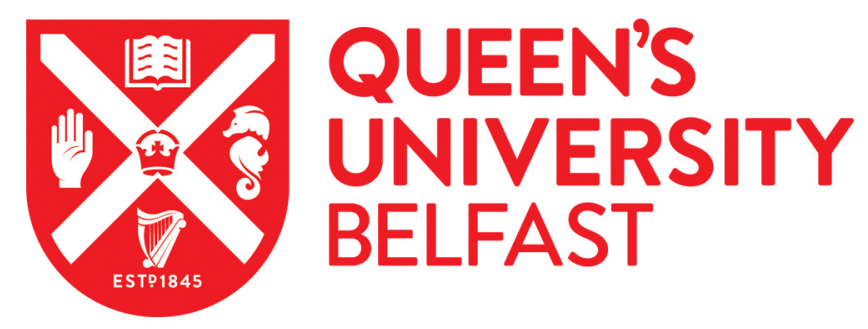

\title{
A BNBF User Selection Scheme for NOMA-Based Cooperative Relaying Systems with SWIPT
}

Do, N. T., Benevides da Cost, D., Duong, T. Q., \& An, B. (2016). A BNBF User Selection Scheme for NOMABased Cooperative Relaying Systems with SWIPT. IEEE Communications Letters.

https://doi.org/10.1109/LCOMM.2016.2631606

\section{Published in:}

IEEE Communications Letters

\section{Document Version:}

Peer reviewed version

\section{Queen's University Belfast - Research Portal:}

Link to publication record in Queen's University Belfast Research Portal

\begin{abstract}
Publisher rights
Copyright 2016 IEEE. Personal use of this material is permitted. Permission from IEEE must be obtained for all other users, including reprinting/ republishing this material for advertising or promotional purposes, creating new collective works for resale or redistribution to servers or lists, or reuse of any copyrighted components of this work in other works.
\end{abstract}

\section{General rights}

Copyright for the publications made accessible via the Queen's University Belfast Research Portal is retained by the author(s) and / or other copyright owners and it is a condition of accessing these publications that users recognise and abide by the legal requirements associated with these rights.

Take down policy

The Research Portal is Queen's institutional repository that provides access to Queen's research output. Every effort has been made to ensure that content in the Research Portal does not infringe any person's rights, or applicable UK laws. If you discover content in the Research Portal that you believe breaches copyright or violates any law, please contact openaccess@qub.ac.uk. 


\title{
A BNBF User Selection Scheme for NOMA-Based Cooperative Relaying Systems with SWIPT
}

\author{
Nhu Tri Do, Student Member, IEEE, Daniel Benevides da Costa, Senior Member, IEEE, \\ Trung Q. Duong, Senior Member, IEEE, and Beongku An, Member, IEEE.
}

\begin{abstract}
In this letter, we investigate the outage performance of cooperative relaying transmissions in two-user non-orthogonal multiple access (NOMA) systems, wherein simultaneous wireless information and power transfer (SWIPT) is employed at the near users to power their relaying operations. To this end, a best-near best-far (BNBF) user selection scheme is proposed. Considering three relaying protocols, i.e., decode-and-forward (DF), amplifyand-forward (AF), and hybrid DF/AF protocols, tight closedform approximate expressions for the outage probability (OP) are derived to evaluate the system performance. Numerical results reveal that, for any relaying protocols used, the diversity order achieved by the BNBF scheme is $M+1$, where $M$ is the number of far users, and does not depend on the number of near users.
\end{abstract}

Index Terms-Non-orthogonal multiple access (NOMA); outage performance; simultaneous wireless information and power transfer (SWIPT); user selection.

\section{INTRODUCTION}

Non-orthogonal multiple access (NOMA) has been considered as an emerging technology for the next generation of cellular networks (i.e., 5G) to improve spectral efficiency by superposing multiple users in power domain [1].

Considering a downlink scenario of a two-user NOMA network that consists of multiple near and far users, it is widely known that in a two-user NOMA system, far users have poorer channel conditions compared to that of near users. As reported in [2], [3], opportunistic scheduling, i.e., the source communicates with only one best destination with the assistance of only one best relay, has been recognized as an attractive scheduling method to improve system performance of such multiuser cooperative network since the time-varying nature of wireless channels is exploited. Owing to this fact, in this paper we adopt the concept of opportunistic scheduling to the two-user NOMA system, where the near users play the role of relays. To this end, a best-near best-far user selection

N. T. Do is with the Department of Electronics and Computer Engineering, Hongik University, Sejong 30016, Korea (email: dotrinhu@gmail.com).

D. B. da Costa is with the Department of Computer Engineering, Federal University of Ceará, Sobral, CE, Brazil (email: danielbcosta@ieee.org).

T. Q. Duong is with the Department of Electronics, Electrical Engineering and Computer Science, Queens University Belfast, Belfast BT7 1NN, U.K (e-mail: trung.q.duong@qub.ac.uk).

B. An is with the Department of Computer and Information Communications Engineering, Hongik University, Sejong 30016, Korea (email: beongku@hongik.ac.kr).

The work of N. T. Do and B. An was supported by the Leading Human Resource Training Program of Regional Neo industry through the Nationa Research Foundation of Korea (NRF) funded by the Ministry of Science, ICT and future Planning (Grant No. 2016H1D5A1910577). The work of D. B. da Costa was supported by the CNPq under Grant 304301/2014-0. Th work of T. Q. Duong was supported in part by the U.K. Royal Academy of Engineering Research Fellowship under Grant RF1415 \14\22. scheme is proposed. Additionally, three relaying protocols, i.e., decode-and-forward (DF), amplify-and-forward (AF), and hybrid DF/AF protocols, are considered.

However, an interesting question that arises is how to perform the cooperative relaying operation without draining the near users' batteries. Fortunately, simultaneous wireless information and power transfer (SWIPT) technique has arisen as a promising and sustainable solution for cooperative relaying in NOMA system, which was reported in several works, such as [1], [4], [5]. This is the motivation to investigate the outage performance of the cooperative two-user NOMA system with SWIPT being employed at the near users.

In our analysis, tight closed-form approximate expressions for the outage probability (OP) of the selected near and far users are obtained. Defining $N$ and $M$ as the numbers of near and far users, respectively, our results reveal that the diversity order achieved by the BNBF scheme for the selected near user is $N$; while that for the selected far user is $M+1$.

\section{System ModeL}

Consider a cooperative network composed of one source, $\mathrm{S}$, a cluster of $M$ far users, $\mathrm{F}=\left\{\mathrm{F}_{j}, j=1, \ldots, M\right\}$, and a cluster of $N$ near users, $\mathrm{N}=\left\{\mathrm{N}_{i}, i=1, \ldots, N\right\}$. We assume that all nodes are equipped with single antenna and operate in half-duplex mode. All wireless links are assumed to undergo independent and identically distributed (i.i.d.) Rayleigh block flat fading. Let $h_{\mathrm{XY}}$ and $\left|h_{\mathrm{XY}}\right|^{2}$ denote the channel coefficient and the corresponding channel gain, respectively, of $\mathrm{X} \rightarrow \mathrm{Y}$ channel; let $w_{\mathrm{Y}}$ denote the additive white Gaussian noise (AWGN) at node $\mathrm{Y}$, where $\mathrm{X} \in\{\mathrm{S}\} \cup \mathrm{N}, \mathrm{Y} \in \mathrm{N} \cup \mathrm{F}$. Without loss of generality, we assume that all terminals have the same AWGN mean power $N_{0}$. We also assume that the source perfectly knows the channel state information (CSI) of all near and far users, as in [4].

\section{A. The Best-Near Best-Far (BNBF) User Selection Scheme}

The proposed user selection process is conducted before data transmission through the signaling and channel state information estimation/calculation system. Specifically, a near user $\mathrm{N}_{\mathrm{s}} \in \mathrm{N}$ and a far user $\mathrm{F}_{\mathrm{s}} \in \mathrm{F}$ that have the best respective channel conditions will be selected in each transmission slot. Mathematically, the BNBF selection criterion can be described as $\mathrm{N}_{\mathrm{s}}=\arg \max _{i=1, \ldots, N}\left|h_{\mathrm{SN}_{i}}\right|^{2}, \mathrm{~F}_{\mathrm{s}}=$ $\arg \max _{j=1, \ldots, M}\left|h_{\mathrm{SF}_{j}}\right|^{2}$. 


\section{B. The NOMA-based Cooperative Transmission with SWIPT}

In this paper, a two-user NOMA scheme is employed in a cooperative relaying downlink transmission, which consists of two phases, each phase has the same duration of $T$ [4].

1) The first phase (direct transmission): Following the principle of NOMA, messages $x_{\mathrm{N}_{\mathrm{s}}}$ and $x_{\mathrm{F}_{\mathrm{s}}}$ that will be transmitted to $\mathrm{N}_{\mathrm{s}}$ and $\mathrm{F}_{\mathrm{s}}$, respectively, are superimposed as $\sqrt{\theta_{\mathrm{N}_{\mathrm{s}}}} x_{\mathrm{N}_{\mathrm{s}}}+\sqrt{\theta_{\mathrm{F}_{\mathrm{s}}}} x_{\mathrm{F}_{\mathrm{s}}}$ and then broadcasted by the source, where $\theta_{\mathrm{N}_{\mathrm{s}}}$ and $\theta_{\mathrm{F}_{\mathrm{s}}}$ are the power allocation coefficients. We assume that $\left|h_{\mathrm{SN}_{i}}\right|^{2}>\left|h_{\mathrm{SF}_{j}}\right|^{2}$, and set $0<\theta_{\mathrm{N}_{\mathrm{s}}}<\theta_{\mathrm{F}_{\mathrm{s}}}$ and $\theta_{\mathrm{N}_{\mathrm{s}}}+\theta_{\mathrm{F}_{\mathrm{s}}}=1$. Thus, the received signal at $\mathrm{F}_{\mathrm{s}}$ from $\mathrm{S}$ can be given by $y_{\mathrm{SF}_{\mathrm{s}}}=\left(\sqrt{\theta_{\mathrm{N}_{\mathrm{s}}} P_{\mathrm{S}}} x_{\mathrm{N}_{\mathrm{s}}}+\sqrt{\theta_{\mathrm{F}_{\mathrm{s}}} P_{\mathrm{S}}} x_{\mathrm{F}_{\mathrm{s}}}\right) h_{\mathrm{SF}_{\mathrm{s}}}+w_{\mathrm{F}_{\mathrm{s}}}$, where $P_{\mathrm{S}}$ denotes the transmit power of the source.

At the far user, the received signal-to-interference-plusnoise ratio (SINR) at $\mathrm{F}_{\mathrm{S}}$ to detect $x_{\mathrm{F}_{\mathrm{s}}}$ transmitted from $\mathrm{S}$ can be written as

$$
\gamma_{\mathrm{SF}_{\mathrm{s}}}=\theta_{\mathrm{F}_{\mathrm{s}}} \bar{\gamma}\left|h_{\mathrm{SF}_{\mathrm{s}}}\right|^{2} /\left(\theta_{\mathrm{N}_{\mathrm{s}}} \bar{\gamma}\left|h_{\mathrm{SF}_{\mathrm{s}}}\right|^{2}+1\right),
$$

where $\bar{\gamma} \triangleq \frac{P_{\mathrm{S}}}{N_{0}}$ denotes the transmit signal-to-noise ratio (SNR).

At the near user, considering a static power-splitting receiver at $\mathrm{N}_{\mathrm{s}}$ to harvest energy from received observations, herein, we further assume that the power splitting receiver only utilizes the signal power, but not the antenna noise power, as done in [6], the energy harvested by $\mathrm{N}_{\mathrm{S}}$ can be expressed as $E_{\mathrm{N}_{\mathrm{s}}}=\rho \eta P_{\mathrm{S}}\left|h_{\mathrm{SN}_{\mathrm{s}}}\right|^{2} T$, where $0<\rho<1$ denoting the power-splitting ratio and $0<\eta<1$ denotes the energy conversion efficiency. Thus, the received signal at $\mathrm{N}_{\mathrm{s}}$ transmitted by $\mathrm{S}$ using NOMA can be given by $y_{\mathrm{SN}_{\mathrm{s}}}=$ $\sqrt{(1-\rho)}\left(\sqrt{\theta_{\mathrm{N}_{\mathrm{s}}} P_{\mathrm{S}}} x_{\mathrm{N}_{\mathrm{s}}}+\sqrt{\theta_{\mathrm{F}_{\mathrm{s}}} P_{\mathrm{S}}} x_{\mathrm{F}_{\mathrm{s}}}\right) h_{\mathrm{SN}_{\mathrm{s}}}+w_{\mathrm{N}_{\mathrm{s}}}$. Note that we only consider the power-splitting receiver architecture in order to alleviate the complexity of cooperative relaying time frame structure. However, the analysis framework presented in this paper can be applied for the time-switching receiver architecture.

Adopting a successive interference cancellation (SIC) receiver [4], $\mathrm{N}_{\mathrm{s}}$ first decodes $x_{\mathrm{F}_{\mathrm{s}}}$ and then subtracts this component from the received signal to detect its own message, $x_{\mathrm{N}_{\mathrm{s}}}$. Thus, the received SINR at $\mathrm{N}_{\mathrm{s}}$ to detect $x_{\mathrm{F}_{\mathrm{s}}}$ can be written as

$$
\gamma_{\mathrm{SN}_{\mathrm{s}}}^{x_{\mathrm{F}_{\mathrm{s}}}}=(1-\rho) \theta_{\mathrm{F}_{\mathrm{s}}} \bar{\gamma}\left|h_{\mathrm{SN}_{\mathrm{s}}}\right|^{2} /\left[(1-\rho) \theta_{\mathrm{N}_{\mathrm{s}}} \bar{\gamma}\left|h_{\mathrm{SN}_{\mathrm{s}}}\right|^{2}+1\right],
$$

and the received SNR at $\mathrm{N}_{\mathrm{S}}$ to detect $x_{\mathrm{N}_{\mathrm{s}}}$ is given by

$$
\gamma_{\mathrm{SN}_{\mathrm{s}}}^{x_{\mathrm{N}_{\mathrm{s}}}}=(1-\rho) \theta_{\mathrm{N}_{\mathrm{s}}} \bar{\gamma}\left|h_{\mathrm{SN}_{\mathrm{s}}}\right|^{2}
$$

2) The second phase (relaying transmission): Assuming that all the harvested energy is used, the transmit power of $\mathrm{N}_{\mathrm{s}}$ can thus be given by $P_{\mathrm{N}_{\mathrm{s}}}=E_{\mathrm{N}_{\mathrm{s}}} / T=\rho \eta P_{\mathrm{S}}\left|h_{\mathrm{SN}_{\mathrm{s}}}\right|^{2}$.

Assuming that DF protocol is adopted at $\mathrm{N}_{\mathrm{s}}$, the received signal at $\mathrm{F}_{\mathrm{S}}$ from $\mathrm{N}_{\mathrm{S}}$ can be expressed as $y_{\mathrm{N}_{\mathrm{s}} \mathrm{F}_{\mathrm{s}}}^{\mathrm{DF}}=$ $P_{\mathrm{N}_{\mathrm{s}}} h_{\mathrm{N}_{\mathrm{s}} \mathrm{F}_{\mathrm{s}}} x_{\mathrm{F}_{\mathrm{s}}}+w_{\mathrm{F}_{\mathrm{s}}}$. Consequently, the received SNR at $\mathrm{F}_{\mathrm{s}}$ to detect $x_{\mathrm{F}_{\mathrm{s}}}$ transmitted by $\mathrm{N}_{\mathrm{s}}$ can be written as

$$
\gamma_{\mathrm{N}_{\mathrm{s}} \mathrm{F}_{\mathrm{s}}}^{\mathrm{DF}}=\rho \eta \bar{\gamma}\left|h_{\mathrm{SN}_{\mathrm{s}}}\right|^{2}\left|h_{\mathrm{N}_{\mathrm{s}} \mathrm{F}_{\mathrm{s}}}\right|^{2}
$$

Assuming variable-gain AF protocol is adopted at $\mathrm{N}_{\mathrm{S}}$ with an amplification factor $G^{2}=\frac{P_{\mathrm{N}_{\mathrm{s}}}}{P_{\mathrm{S}}\left|h_{\mathrm{SN}_{\mathrm{s}}}\right|^{2}+N_{0}}=\frac{\rho \eta \bar{\gamma}\left|h_{\mathrm{SN}_{\mathrm{s}}}\right|^{2}}{\bar{\gamma}\left|h_{\mathrm{SN}_{\mathrm{s}}}\right|^{2}+1}$, the received SINR at $\mathrm{F}_{\mathrm{S}}$ to detect $x_{\mathrm{F}_{\mathrm{s}}}$ transmitted from $\mathrm{N}_{\mathrm{S}}$ using AF protocol can be given by

$$
\begin{aligned}
& \gamma_{\mathrm{N}_{\mathrm{s}} \mathrm{F}_{\mathrm{s}}}^{\mathrm{AF}}=\frac{\rho(1-\rho) \eta \bar{\gamma}^{2} \theta_{\mathrm{F}_{\mathrm{s}}}\left|h_{\mathrm{SN}_{\mathrm{s}}}\right|^{4}\left|h_{\mathrm{N}_{\mathrm{s}} \mathrm{F}_{\mathrm{s}}}\right|^{2}}{\left[\rho(1-\rho) \eta \bar{\gamma}^{2} \theta_{\mathrm{N}_{\mathrm{s}}}\left|h_{\mathrm{SN}_{\mathrm{s}}}\right|^{4}\left|h_{\mathrm{N}_{\mathrm{s}} \mathrm{F}_{\mathrm{s}}}\right|^{2}\right.} . \\
& \left.+\rho \eta \bar{\gamma}\left|h_{\mathrm{SN}_{\mathrm{s}}}\right|^{2}\left|h_{\mathrm{N}_{\mathrm{s}} \mathrm{F}_{\mathrm{s}}}\right|^{2}+\bar{\gamma}\left|h_{\mathrm{SN}_{\mathrm{s}}}\right|^{2}+1\right]
\end{aligned}
$$

It is noteworthy that AF protocol can be employed even if $\mathrm{N}_{\mathrm{S}}$ fails to decode $x_{\mathrm{F}_{\mathrm{s}}}$.

Assuming hybrid protocol is adopted at $\mathrm{N}_{\mathrm{s}}$, if $\mathrm{N}_{\mathrm{s}}$ successfully decodes $x_{\mathrm{F}_{\mathrm{s}}}$, then DF protocol will be used to forward $x_{\mathrm{F}_{\mathrm{s}}}$ to $\mathrm{F}_{\mathrm{s}}$, otherwise, AF protocol will be used.

Finally, $\mathrm{F}_{\mathrm{s}}$ combines two signals, i.e., the direct signal from $\mathrm{S}$ and the relaying signal from $\mathrm{N}_{\mathrm{S}}$ using selection combining (SC) technique.

\section{Performance AnAlysis}

Considering i.i.d. Rayleigh channels, $\left|h_{\mathrm{SN}_{i}}\right|^{2},\left|h_{\mathrm{SF}_{j}}\right|^{2}$, and $\left|h_{\mathrm{N}_{i} \mathrm{~F}_{j}}\right|^{2}$, where $i=1, \ldots, N, j=1, \ldots, M$, follow exponential distributions with parameters $\lambda_{\mathrm{SN}}=d_{\mathrm{SN}}^{\epsilon}, \lambda_{\mathrm{SF}}=d_{\mathrm{SF}}^{\epsilon}$, $\lambda_{\mathrm{NF}}=d_{\mathrm{NF}}^{\epsilon}$, respectively, where $d$ and $\epsilon$ denote the Euclidean distance and path-loss exponent, respectively.

Let $X \triangleq\left|h_{\mathrm{SN}_{\mathrm{s}}}\right|^{2}, Y \triangleq\left|h_{\mathrm{SF}_{\mathrm{s}}}\right|^{2}$, and $V \in\{X, Y\}$. The cumulative distribution function $(\mathrm{CDF}), F_{V}(z)$, and the probability density function (PDF), $f_{V}(v)$, are written as $F_{V}(v)=\sum_{k=0}^{K}\left(\begin{array}{c}K \\ k\end{array}\right)(-1)^{k} e^{-k \lambda_{V} v}$, and $f_{V}(v)=$ $\sum_{k=1}^{K}\left(\begin{array}{c}K \\ k\end{array}\right)(-1)^{k+1} k \lambda_{V} e^{-k \lambda_{V} v}$, respectively, where $K \in$ $\{M, N\}, \lambda_{V} \in\left\{\lambda_{\mathrm{SF}}, \lambda_{\mathrm{SN}}\right\}$. Regarding the CDF and PDF of $V$, these are the order statistics for the maximum of the i.i.d. channel gains. However it is implied that the destinations can be located in a relatively small area, and so do the relays.

Let $R_{1}$ and $R_{2}$ (bits/s/Hz) denote target data rates of $\mathrm{N}_{\mathrm{s}}$ and $\mathrm{F}_{\mathrm{S}}$, respectively. Consequently, $\gamma_{i}=2^{2 R_{i}}-1, i=$ 1,2 are the SNR thresholds for correctly decoding $x_{\mathrm{N}_{\mathrm{s}}}$ and $x_{\mathrm{F}_{\mathrm{s}}}$, respectively. And let $\gamma_{\mathrm{e} 2 \mathrm{e}, \mathrm{F}_{\mathrm{s}}}^{\mathrm{DF}}=\max \left\{\gamma_{\mathrm{SF}_{\mathrm{s}}}, \gamma_{\mathrm{N}_{\mathrm{s}} \mathrm{F}_{\mathrm{s}}}^{\mathrm{DF}}\right\}$ and $\gamma_{\mathrm{e} 2 \mathrm{e}, \mathrm{F}_{\mathrm{s}}}^{\mathrm{AF}}=\max \left\{\gamma_{\mathrm{SF}_{\mathrm{s}}}, \gamma_{\mathrm{N}_{\mathrm{s}} \mathrm{F}_{\mathrm{s}}}^{\mathrm{AF}}\right\}$ denote the end-to-end SNR of the selected far user $\mathrm{F}_{\mathrm{S}}$ in the case of using DF and AF protocols, respectively.

\section{A. Outage Probability $(\mathrm{OP})$ of The Selected Far User $\mathrm{F}_{\mathrm{s}}$}

1) DF Protocol: The OP of $\mathrm{F}_{\mathrm{S}}$ can be expressed as

$$
\begin{aligned}
P_{\mathrm{out}, \mathrm{F}_{\mathrm{s}}}^{\mathrm{DF}}=\operatorname{Pr} & \left(\gamma_{\mathrm{SN}_{\mathrm{s}}}^{x_{\mathrm{F}_{\mathrm{s}}}}<\gamma_{2}, \gamma_{\mathrm{SF}_{\mathrm{s}}}<\gamma_{2}\right) \\
& +\operatorname{Pr}\left(\gamma_{\mathrm{SN}_{\mathrm{s}}}^{x_{\mathrm{F}_{\mathrm{s}}}} \geq \gamma_{2}, \gamma_{\mathrm{e} 2 \mathrm{e}, \mathrm{F}_{\mathrm{s}}}^{\mathrm{DF}}<\gamma_{2}\right) .
\end{aligned}
$$

Let $\theta_{\mathrm{s}} \triangleq \theta_{\mathrm{F}_{\mathrm{s}}} / \theta_{\mathrm{N}_{\mathrm{s}}}$. The first term of the right-hand side of (6) can be obtained as

$$
\Phi_{1}=\operatorname{Pr}\left(\gamma_{\mathrm{SN}_{\mathrm{s}}}^{x_{\mathrm{F}_{\mathrm{s}}}}<\gamma_{2}\right) \operatorname{Pr}\left(\gamma_{\mathrm{SF}_{\mathrm{s}}}<\gamma_{2}\right)=\Phi_{1 A} \Phi_{1 B},
$$

where

$$
\Phi_{1 A}=\operatorname{Pr}\left(\frac{a_{1} X}{a_{2} X+1}<\gamma_{2}\right)=\sum_{k=0}^{N}\left(\begin{array}{c}
N \\
k
\end{array}\right)(-1)^{k} e^{-\frac{k \lambda_{\mathrm{SN}} \gamma_{2}}{a_{1}-a_{2} \gamma_{2}}},
$$

if $\gamma_{2}<a_{1} / a_{2}=\theta_{\mathrm{s}}$, otherwise, $\Phi_{1}=1$, where $a_{1} \triangleq(1-$ $\rho) \theta_{\mathrm{F}_{\mathrm{s}}} \bar{\gamma}, a_{2} \triangleq(1-\rho) \theta_{\mathrm{N}_{\mathrm{s}}} \bar{\gamma}$; and

$$
\Phi_{1 B}=\operatorname{Pr}\left(\frac{b_{1} Y}{b_{2} Y+1}<\gamma_{2}\right)=\sum_{k=0}^{M}\left(\begin{array}{c}
M \\
k
\end{array}\right)(-1)^{k} e^{-\frac{k \lambda_{\mathrm{SF}} \gamma_{2}}{b_{1}-b_{2} \gamma_{2}}} .
$$


if $\gamma_{2}<b_{1} / b_{2}=\theta_{\mathrm{s}}$, otherwise, $\Phi_{2 A}=1$, where $b_{1} \triangleq \theta_{\mathrm{F}_{\mathrm{s}}} \bar{\gamma}$, $b_{2} \triangleq \theta_{\mathrm{N}_{\mathrm{s}}} \bar{\gamma}$.

The second term of the right-hand side of (6) can be reexpressed as

$$
\Phi_{2}=\operatorname{Pr}\left(\gamma_{\mathrm{SF}_{\mathrm{s}}}<\gamma_{2}\right) \operatorname{Pr}\left(\gamma_{\mathrm{SN}_{\mathrm{s}}}^{x_{\mathrm{F}_{\mathrm{s}}}} \geq \gamma_{2}, \gamma_{\mathrm{N}_{\mathrm{s}} \mathrm{F}_{\mathrm{s}}}^{\mathrm{DF}}<\gamma_{2}\right)=\Phi_{1 B} \Phi_{2 A},
$$

where

$$
\Phi_{2 A}=\operatorname{Pr}\left(\frac{a_{1} X}{a_{2} X+1} \geq \gamma_{2}, c X Z<\gamma_{2}\right),
$$

where $c \triangleq \rho \eta \bar{\gamma}$, and $Z \triangleq\left|h_{\mathrm{N}_{\mathrm{s}} \mathrm{F}_{\mathrm{s}}}\right|^{2}$. Considering i.i.d. channels, we have $\operatorname{Pr}\left(\mathrm{N}_{\mathrm{s}}=\mathrm{N}_{i}\right)=1 / N$ and $\operatorname{Pr}\left(\mathrm{F}_{\mathrm{s}}=\mathrm{F}_{j}\right)=1 / M$. Thus, $F_{\left|h_{\mathrm{N}_{\mathrm{s}} \mathrm{F}_{\mathrm{s}}}\right|^{2}}(z)=\sum_{m=1}^{M} \sum_{n=1}^{N} \frac{1}{M N} F_{\left|h_{\mathrm{N}_{i} \mathrm{~F}_{j}}\right|^{2}}(z)$. Consequently, $F_{Z}(z)=1-e^{-\lambda_{\mathrm{NF}} z}$. It can be observed that $\Phi_{2 A}=0$ if $\gamma_{2} \geq \theta_{\mathrm{s}}$. For the case $\gamma_{2}<\theta_{\mathrm{s}}$, conditioning on $X=x, \Phi_{2 A}$ can be rewritten as

$$
\begin{aligned}
\Phi_{2 A} & =\int_{0}^{\infty} \operatorname{Pr}\left(\frac{a_{1} x}{a_{2} x+1} \geq \gamma_{2}, c x Z<\gamma_{2}\right) f_{X}(x) d x, \\
& =\int_{\mu}^{\infty} F_{Z}\left(\frac{\gamma_{2}}{c x}\right) f_{X}(x) d x=\Phi_{2 A_{1}}-\Phi_{2 A_{2}},
\end{aligned}
$$

where $\mu \triangleq \gamma_{2} /\left(a_{1}-a_{2} \gamma_{2}\right)$, and $\Phi_{2 A_{1}}$ is obtained as $\Phi_{2 A_{1}}=$ $\sum_{k=1}^{N}\left(\begin{array}{l}N \\ k\end{array}\right)(-1)^{k+1} e^{-k \lambda_{\mathrm{SN}} \mu}$, while $\Phi_{2 A_{2}}$ can be expressed as

$$
\Phi_{2 A_{2}}=\sum_{k=1}^{N}\left(\begin{array}{l}
N \\
k
\end{array}\right)(-1)^{k+1} k \lambda_{\mathrm{SN}} \int_{\mu}^{\infty} e^{-\frac{\lambda_{\mathrm{NF}} \gamma_{2}}{c x}-k \lambda_{\mathrm{SN}} x} d x .
$$

Since the integral in (13) cannot be further simplified, we will make use of the following approximation $e^{-\alpha x} \approx 1-\alpha x$ for small value of $|x|$. Afterwards, by plugging (13) into (12), $\Phi_{2 A}$ can be derived as

$$
\Phi_{2 A}=\sum_{k=1}^{N}\left(\begin{array}{l}
N \\
k
\end{array}\right)(-1)^{k+1} \frac{k \lambda_{\mathrm{SN}} \lambda_{\mathrm{NF}} \gamma_{2}}{c}\left(-\operatorname{Ei}\left(-k \lambda_{\mathrm{SN}} \mu\right)\right),
$$

where $\operatorname{Ei}(\cdot)$ denotes the exponential integral function $[7, \mathrm{Eq}$. (8.211.1)]. Finally, by substituting (9) and (14) into (10), and then combining with (7), $P_{\mathrm{out}, \mathrm{F}_{\mathrm{s}}}^{\mathrm{DF}}$ is attained as

$$
P_{\text {out }, \mathrm{F}_{\mathrm{s}}}^{\mathrm{DF}}=\Phi_{1 B \mid \text { Eq. (9) }}\left(\Phi_{1 A \mid \text { Eq. (8) }}+\Phi_{2 A \mid \text { Eq. (14) }}\right),
$$

when $\gamma_{2}<\theta_{\mathrm{s}}$, otherwise, $P_{\mathrm{out}, \mathrm{F}_{\mathrm{s}}}^{\mathrm{DF}}=1$.

2) AF Protocol: The OP of $\mathrm{F}_{\mathrm{s}}$ can be expressed as

$$
P_{\text {out }, \mathrm{F}_{\mathrm{s}}}^{\mathrm{AF}}=\operatorname{Pr}\left(\gamma_{\mathrm{e} 2 \mathrm{e}, \mathrm{F}_{\mathrm{s}}}^{\mathrm{AF}}<\gamma_{2}\right),
$$

which can be rewritten as

$$
P_{\text {out }, \mathrm{F}_{\mathrm{s}}}^{\mathrm{AF}}=\operatorname{Pr}\left(\gamma_{\mathrm{SF}_{\mathrm{s}}}<\gamma_{2}\right) \operatorname{Pr}\left(\gamma_{\mathrm{N}_{\mathrm{s}} \mathrm{F}_{\mathrm{s}}}^{\mathrm{AF}_{1}}<\gamma_{2}\right)=\Phi_{1 B} \Psi,
$$

where

$$
\Psi=\operatorname{Pr}\left(\frac{a_{1} c X^{2} Z}{a_{2} c X^{2} Z+c X Z+\bar{\gamma} X+1}<\gamma_{2}\right) .
$$

Since it is hard to directly derive (18), we will resort on $\frac{\alpha}{\beta+1} \approx \frac{\alpha}{\beta}$ which holds when $\beta$ is sufficient large. Then, $\Psi$ can be approximated by

$$
\begin{aligned}
\Psi & \approx \operatorname{Pr}\left(\frac{a_{1} c X Z}{a_{2} c X Z+c Z+\bar{\gamma}}<\gamma_{2}\right) \\
& =\int_{0}^{\infty} \operatorname{Pr}\left(\left[\left(a_{1}-a_{2} \gamma_{2}\right) x-\gamma_{2}\right] c Z<\bar{\gamma} \gamma_{2}\right) f_{X}(x) d x
\end{aligned}
$$

As can be observed, $\Phi_{2 A}=\Psi=1$ if $\gamma_{2} \geq \theta_{\mathrm{s}}$. For the case $\gamma_{2}<\theta_{\mathrm{s}}$, conditioning on $X=x, \Psi$ can be expressed as

$$
\begin{aligned}
& \Psi= \int_{0}^{\mu} f_{X}(x)+\int_{\mu}^{\infty} \operatorname{Pr}\left(Z<\frac{\bar{\gamma} \gamma_{2}}{\left[\left(a_{1}-a_{2} \gamma_{2}\right) x-\gamma_{2}\right] c}\right) f_{X}(x) \\
&=1-\sum_{k=1}^{N}\left(\begin{array}{c}
N \\
k
\end{array}\right)(-1)^{k+1} k \lambda_{\mathrm{SN}} \\
& \quad \times \int_{\mu}^{\infty} e^{-\frac{\lambda_{\mathrm{NF}} \bar{\gamma} \gamma_{2}}{\left[\left(a_{1}-a_{2} \gamma_{2}\right) x-\gamma_{2}\right] c}-k \lambda_{\mathrm{SN}} x} d x
\end{aligned}
$$

where $\mu=\gamma_{2} /\left(a_{1}-a_{2} \gamma_{2}\right)$. By using the following change of variables, i.e., $u=\left(a_{1}-a_{2} \gamma_{2}\right) x-\gamma_{2}$, and then applying the formula $\int_{0}^{\infty} e^{-\frac{\beta}{4 x}-\gamma x} d x=\sqrt{\frac{\beta}{\gamma}} K_{1}(\sqrt{\beta \gamma})$ [7, Eq. (3.324.1)], $\Psi$ can be derived as

$$
\Psi=1-\sum_{k=1}^{N}\left(\begin{array}{l}
N \\
k
\end{array}\right)(-1)^{k+1} e^{-k \lambda_{\mathrm{SN}} \mu} \sqrt{\chi} K_{1}(\sqrt{\chi}),
$$

where $\chi=\frac{4 k \lambda_{\mathrm{SN}} \lambda_{\mathrm{NF}} \bar{\gamma} \mu}{c}$, and $K_{1}(\cdot)$ denotes the first-order modified Bessel function of the second kind [7, Eq. (8.407.1)]. By plugging (9) and (21) into (17), $P_{\mathrm{out}, \mathrm{F}_{\mathrm{s}}}^{\mathrm{AF}}$ is attained as

$$
P_{\text {out }, \mathrm{F}_{\mathrm{s}}}^{\mathrm{AF}}=\Phi_{1 B \mid \text { Eq. (9) }} \Psi_{\mid \text {Eq. (21) }},
$$

when $\gamma_{2}<\theta_{\mathrm{s}}$, otherwise, $P_{\mathrm{out}, \mathrm{F}_{\mathrm{s}}}^{\mathrm{AF}}=1$.

3) Hybrid Protocol: The OP of $\mathrm{F}_{\mathrm{S}}$ can be expressed as

$$
\begin{aligned}
P_{\text {out, } \mathrm{F}_{\mathrm{s}}}^{\text {Hybrid }}= & \operatorname{Pr}\left(\gamma_{\mathrm{SN}_{\mathrm{s}}}^{x_{\mathrm{F}_{\mathrm{s}}}}<\gamma_{2}, \max \left\{\gamma_{\mathrm{SF}_{\mathrm{s}}}, \gamma_{\mathrm{N}_{\mathrm{s}} \mathrm{F}_{\mathrm{s}}}^{\mathrm{AF}}\right\}<\gamma_{2}\right) \\
& +\operatorname{Pr}\left(\gamma_{\mathrm{SN}_{\mathrm{s}}}^{x_{\mathrm{F}_{\mathrm{s}}}} \geq \gamma_{2}, \max \left\{\gamma_{\mathrm{SF}_{\mathrm{s}}}, \gamma_{\mathrm{N}_{\mathrm{s}} \mathrm{F}_{\mathrm{s}}}^{\mathrm{DF}}\right\}<\gamma_{2}\right) \\
= & \Phi_{1 B} \Theta+\Phi_{2},
\end{aligned}
$$

where $\Phi_{1 B}$ and $\Phi_{2}$ are presented in (9) and (10), respectively, and $\Theta$ can be derived as

$$
\begin{aligned}
& \Theta=\operatorname{Pr}\left(\frac{a_{1} X}{a_{2} X+1}<\gamma_{2}, \frac{a_{1} c X^{2} Z}{a_{2} c X^{2} Z+c X Z+\bar{\gamma} X+1}<\gamma_{2}\right) \\
&= \operatorname{Pr}\left(a_{1} X-a_{2} \gamma_{2} X-\gamma_{2}<0,\right. \\
&\left.\quad\left(a_{1} X-a_{2} \gamma_{2} X-\gamma_{2}\right) c X Z<\gamma_{2} \bar{\gamma} X+\gamma_{2}\right) .
\end{aligned}
$$

Conditioning on $X=x$, from (24) we can see that $\operatorname{Pr}\left(Z>\frac{\gamma_{2} \bar{\gamma} x+\gamma_{2}}{\left(a_{1} x-a_{2} \gamma_{2} x-\gamma_{2}\right) c x}\right)=1$ since $a_{1} x-a_{2} \gamma_{2} x-\gamma_{2}<0$. Consequently, $\Theta$ can be attained as

$$
\Theta=\int_{0}^{\mu} f_{X}(x) d x=\sum_{k=1}^{N}\left(\begin{array}{l}
N \\
k
\end{array}\right)(-1)^{k+1}\left[1-e^{-\frac{k \lambda_{S N} \gamma_{2}}{a_{1}-a_{2} \gamma_{2}}}\right] .
$$

Thus, the OP of $\mathrm{F}_{\mathrm{S}}$ using hybrid protocol achieved as

$$
P_{\text {out }, \mathrm{F}_{\mathrm{s}}}^{\text {Hybrid }}=\Phi_{1 B \mid \text { Eq. (9) }}\left(\Phi_{2 A \mid \text { Eq. (14) }}+\Theta_{\mid \text {Eq. (25) }}\right),
$$

when $\gamma_{2}<\theta_{\mathrm{s}}$, otherwise, $P_{\text {out }, \mathrm{F}_{\mathrm{s}}}^{\text {Hybrid }}=1$.

\section{B. Outage Probability (OP) of The Selected Near User $\mathrm{N}_{\mathrm{s}}$}

The OP of the selected near user $P_{\text {out }}^{\mathrm{N}_{\mathrm{s}}}$ can be expressed as

$$
P_{\text {out }}^{N_{\mathrm{s}}}=\operatorname{Pr}\left(\gamma_{\mathrm{SN}_{\mathrm{s}}}^{x_{\mathrm{F}_{\mathrm{s}}}}<\gamma_{2}\right)+\operatorname{Pr}\left(\gamma_{\mathrm{SN}_{\mathrm{s}}}^{x_{\mathrm{F}_{\mathrm{s}}}} \geq \gamma_{2}, \gamma_{\mathrm{SN}_{\mathrm{s}}}^{x_{\mathrm{N}_{\mathrm{s}}}}<\gamma_{1}\right) \text {. }
$$

which can be attained as

$$
\begin{aligned}
& P_{\text {out }}^{\mathrm{N}_{\mathrm{s}}}=\sum_{k=0}^{N}\left(\begin{array}{l}
N \\
k
\end{array}\right)(-1)^{k} e^{-k \lambda_{\mathrm{SN}} \gamma_{1} / a_{2}}, \text { if } \gamma_{1}>\mu a_{2}, \gamma_{2}<\theta_{\mathrm{s}} \\
& P_{\mathrm{out}}^{\mathrm{N}_{\mathrm{s}}}=\sum_{k=0}^{N}\left(\begin{array}{c}
N \\
k
\end{array}\right)(-1)^{k} e^{-k \lambda_{\mathrm{SN}} \mu}, \text { if } \gamma_{1} \leq \mu a_{2}, \gamma_{2}<\theta_{\mathrm{s}} ; \text { and } \\
& P_{\mathrm{out}}^{\mathrm{N}_{\mathrm{s}}}=1, \text { if } \gamma_{2} \geq \theta_{\mathrm{s}}, \forall \gamma_{1} .
\end{aligned}
$$




\section{Diversity Analysis of The Selected Far Users $\mathrm{F}_{\mathrm{s}}$}

Using [7, Eq. (1.111)] and the fact that $e^{-\alpha / y} \approx 1-\alpha / y$ when $y \rightarrow \infty$, we have

$$
\begin{aligned}
\Phi_{1 A}^{\text {asym }}= & \Theta^{\text {asym }}=\left[\lambda_{\mathrm{SN}} \gamma_{2} /\left((1-\rho)\left(\theta_{\mathrm{F}_{\mathrm{s}}}-\theta_{\mathrm{N}_{\mathrm{s}}} \gamma_{2}\right) \bar{\gamma}\right)\right]^{N}, \\
\Phi_{1 B}^{\text {asym }} & =\left[\lambda_{\mathrm{SF}} \gamma_{2} /\left(\left(\theta_{\mathrm{F}_{\mathrm{s}}}-\theta_{\mathrm{N}_{\mathrm{s}}} \gamma_{2}\right) \bar{\gamma}\right)\right]^{M} .
\end{aligned}
$$

Also, relying on the fact that $\operatorname{Ei}(-x) \approx C+\ln (x)$ when $x \rightarrow 0^{+}$[7, Eq. (8.214.1)], where $C$ denotes the Euler's constant [7, Eq. (8.367.1)], and the formula $\sum_{k=1}^{N}\left(\begin{array}{l}N \\ k\end{array}\right)(-1)^{k+1} k=0$ [7, Eq. $\left.(0.154 .2)\right], \Phi_{2}$ in (14) can be approximated by

$$
\Phi_{2 A}^{\text {asym }}=-\sum_{k=1}^{N}\left(\begin{array}{c}
N \\
k
\end{array}\right)(-1)^{k+1} \frac{k u_{1}}{\bar{\gamma}} \ln \left(\frac{k u_{2}}{\bar{\gamma}}\right),
$$

where $u_{1}=\frac{\lambda_{\mathrm{SN}} \lambda_{\mathrm{NF}} \gamma_{2}}{\rho \eta}$ and $u_{2}=\frac{\lambda_{\mathrm{SN}} \gamma_{2}}{(1-\rho)\left(\theta_{\mathrm{F}_{\mathrm{s}}}-\theta_{\mathrm{N}_{\mathrm{s}}} \gamma_{2}\right)}$.

On the other hand, using the formula $x K_{1}(x) \approx 1+\frac{x^{2}}{2} \ln \frac{x}{2}$ [8, Eq. (25)] and $\sum_{k=1}^{N}\left(\begin{array}{l}N \\ k\end{array}\right)(-1)^{k+1} k=0, \Theta_{2}$ in (21) can be approximated by

$$
\Psi^{\text {asym }}=-\sum_{k=1}^{N}\left(\begin{array}{l}
N \\
k
\end{array}\right)(-1)^{k+1} \frac{2 k u_{3}}{\bar{\gamma}} \ln \left(\sqrt{\frac{k u_{3}}{\bar{\gamma}}}\right),
$$

where $u_{3}=\lambda_{\mathrm{SN}} \lambda_{\mathrm{NF}} \gamma_{2} /\left[\rho \eta(1-\rho)\left(\theta_{\mathrm{F}_{\mathrm{s}}}-\theta_{\mathrm{N}_{\mathrm{s}}} \gamma_{2}\right)\right]$

From (28), (29), (30), and (31), the asymptotic OPs of $F_{\mathrm{s}}$ using DF, AF, and hybrid DF/AF protocols can be obtained.

Since $\lim _{x \rightarrow \infty} \frac{\log \left[-\frac{\alpha}{x^{M+1}} \ln \left(\frac{\beta}{x^{\kappa}}\right)\right]}{\log x}=-(M+1)$, where $\kappa=1 / 2$ or 1 , and $\sum_{k=1}^{N}\left(\begin{array}{l}N \\ k\end{array}\right)(-1)^{k+1}=1$, it can be concluded that the diversity order achieved by the BNBF scheme at $\mathrm{F}_{\mathrm{s}}$ is $M+1$.

\section{Diversity Analysis of The Selected Near Users $\mathrm{N}_{\mathrm{s}}$}

Similar to (28), the asymptotic OP of $\mathrm{N}_{\mathrm{S}}$ is obtained as: $P_{\mathrm{asym}}^{\mathrm{N}_{\mathrm{s}}}=\left(\lambda_{\mathrm{SN}} \gamma_{1} /(1-\rho) / \theta_{\mathrm{N}_{\mathrm{s}}} / \bar{\gamma}\right)^{N}$ if $\gamma_{1}>\mu a_{2}, \gamma_{2}<\theta_{\mathrm{s}}$, $P_{\mathrm{asym}}^{\mathrm{N}_{\mathrm{s}}}=\left(\lambda_{\mathrm{SN}} \gamma_{2} /(1-\rho) /\left(\theta_{\mathrm{F}_{\mathrm{s}}}-\theta_{\mathrm{N}_{\mathrm{s}}} \gamma_{2}\right) / \bar{\gamma}\right)^{N}$ if $\gamma_{1} \leq \mu a_{2}$, $\gamma_{2}<\theta_{\mathrm{s}}$, otherwise, $P_{\text {out, asym }}^{\mathrm{N}_{\mathrm{s}}}=1$. Consequently, the diversity order achieved by the BNBF selection scheme for $\mathrm{N}_{\mathrm{S}}$ is $N$.

\section{NUMERICAL RESULTS AND DISCUSSIONS}

In this Section, we set $\theta_{\mathrm{N}_{\mathrm{s}}}=1 / 5, \theta_{\mathrm{F}_{\mathrm{s}}}=4 / 5$; and $\epsilon=$ $3, \eta=0.7, \rho=0.5$, the target data rates $R_{1}=R_{2}=1$ (bits/s/Hz), the coordinates of source, near users, and far users are $(0,0.5),(0.2,0.5)$, and $(1,0.5)$, respectively.

As shown in Fig. 1, the hybrid DF/AF protocol and the DF protocol have similar performance and are better than that of the AF protocol. This can be explained that, in (24), $\operatorname{Pr}\left(\frac{a_{1} c x^{2} Z}{a_{2} c x^{2} Z+c x Z+\bar{\gamma} x+1}<\gamma_{2}\right)=1$ when $\frac{a_{1} x}{a_{2} x+1}<\gamma_{2}$ means that when $\mathrm{N}_{\mathrm{s}}$ fails to decode $x_{\mathrm{F}_{\mathrm{s}}}$, its amplifying and forwarding signal does not help to improve the reliability of $\mathrm{F}_{\mathrm{s}}$. As can be observed, based on the slopes of performance curves at high SNR regime, for any relaying protocols used, the diversity oder achieved by the BNBF scheme at the far user is the same and higher than that achieved at the near user.

In Fig. 2, we compare the performance of the BNBF scheme with that of best-near worst-far (BNWF) scheme, worstnear best-far (WNBF) scheme, and random-near random-far

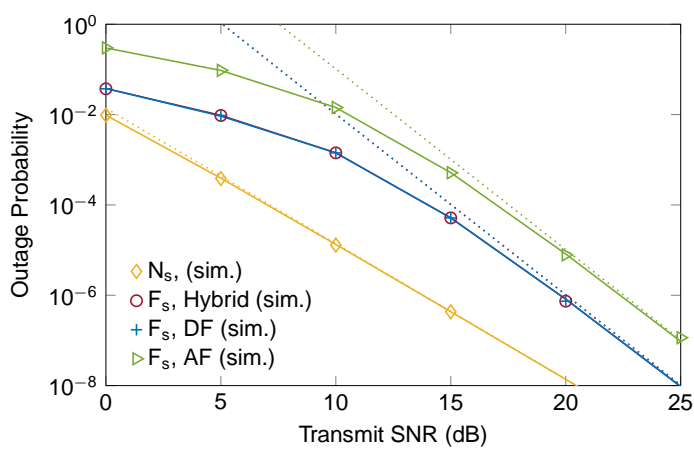

Fig. 1. Outage probability and asymptotic OP of $\mathrm{N}_{\mathrm{S}}$ and $\mathrm{F}_{\mathrm{S}}$ as a function of transmit SNR. Solid lines and dotted lines represent approximate results and asymptotic results, respectively.

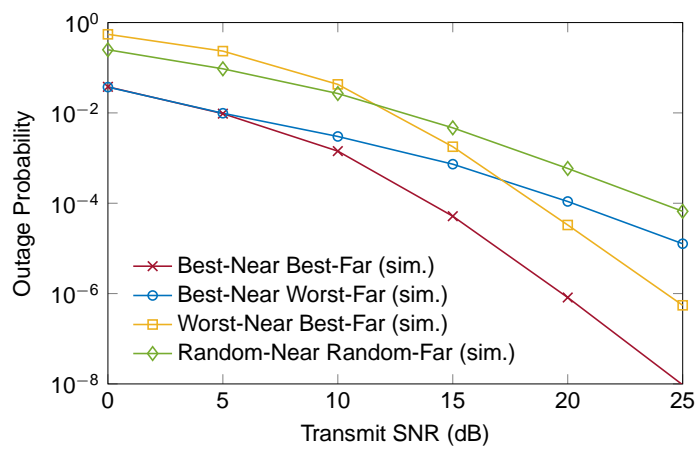

Fig. 2. Performance comparison between BNBF, BNWF, WNBF, and RNRF schemes.

(RNRF) scheme using computer simulation. As can be seen, the outage performance of the BNBF scheme is the best. Also note that the diversity order of BNBF scheme is higher than that of BNWF and RNRF schemes and is similar to that of WNBF scheme. This is consistent with our analysis, i.e., the diversity order achieved by the BNBF scheme is $M+1$, and does not depend on the number of near users $N$.

\section{REFERENCES}

[1] Z. Ding, Y. Liu, J. Choi, Q. Sun, M. Elkashlan, and H. V. Poor "Application of non-orthogonal multiple access in LTE and 5G networks." [Online]. Available: http://arxiv.org/abs/1511.08610

[2] N. Yang, M. Elkashlan, and J. Yuan, "Outage probability of multiuser relay networks in nakagami- $m$ fading channels," IEEE Trans. Veh. Technol., vol. 59, no. 5, pp. 2120-2132, Jun. 2010.

[3] K. Elkhalil, M. E. Eltayeb, A. Kammoun, T. Y. Al-Naffouri, and H. R. Bahrami, "On the feedback reduction of multiuser relay networks using compressive sensing," IEEE Trans. Wireless Commun., vol. 64, no. 4, pp. 1437-1450, Apr. 2016.

[4] Y. Liu, Z. Ding, M. Elkashlan, and H. V. Poor, "Cooperative nonorthogonal multiple access with simultaneous wireless information and power transfer," IEEE J. Select. Areas Commun., vol. 34, no. 4, pp. 938953, Apr. 2016.

[5] P. Diamantoulakis, K. Pappi, Z. Ding, and G. Karagiannidis, "Wireless powered communications with non-orthogonal multiple access," IEEE Trans. Wireless Commun., 2016 (to be appeared).

[6] L. Liu, R. Zhang, and K. C. Chua, "Wireless information and power transfer: a dynamic power splitting approach," IEEE Trans. Commun., vol. 61, no. 9, pp. 3990-4001, Sep. 2013.

[7] I. S. Gradshteyn and I. M. Ryzhik, Tables of Integrals, Series, and Products 7th Ed. New York: Academic Press, 2007.

[8] Z. Ding, S. M. Perlaza, I. Esnaola, and H. V. Poor, "Power allocation strategies in energy harvesting wireless cooperative networks," IEEE Trans. Wireless Commun., vol. 13, no. 2, pp. 846-860, Feb. 2014. 